BNL-114327-2017-JA

\title{
Experimental Evidence of Ion-induced Instabilities in the NSLS-II Storage Ring
}

\author{
W. Cheng, Y. Li \\ Accepted in Nuclear Instruments and Methods in Physics Research Section A: \\ Accelerators, Spectrometers, Detectors and Associated Equipment
}

March 2017

Photon Sciences Department

Brookhaven National Laboratory

\author{
U.S. Department of Energy \\ USDOE Office of Science (SC), \\ Basic Energy Sciences (BES) (SC-22)
}




\section{DISCLAIMER}

This report was prepared as an account of work sponsored by an agency of the United States Government. Neither the United States Government nor any agency thereof, nor any of their employees, nor any of their contractors, subcontractors, or their employees, makes any warranty, express or implied, or assumes any legal liability or responsibility for the accuracy, completeness, or any third party's use or the results of such use of any information, apparatus, product, or process disclosed, or represents that its use would not infringe privately owned rights. Reference herein to any specific commercial product, process, or service by trade name, trademark, manufacturer, or otherwise, does not necessarily constitute or imply its endorsement, recommendation, or favoring by the United States Government or any agency thereof or its contractors or subcontractors. The views and opinions of authors expressed herein do not necessarily state or reflect those of the United States Government or any agency thereof. 


\section{Experimental Evidence of Ion-induced Instabilities in the NSLS-II Storage Ring*

\author{
Weixing Cheng", Yongjun Li, Boris Podobedov
} \\ NSLS-II, Brookhaven National Laboratory, Upton, NY 11973}

\section{Abstract}

Fast ion instability has been identified as one of the most prominent instabilities in the recently constructed NSLS-II storage ring at Brookhaven National Laboratory. At a relatively low beam current $(\sim 25 \mathrm{~mA})$ multi-bunch fills, ion-induced instabilities have already been observed during the early stages of machine commissioning. At present user operation with $250 \mathrm{~mA}$ in $\sim 1000$ bunches, the fast ion still remains the dominant instability, even after months of vacuum conditioning at high current. Ioninduced dipole motions of the electron beam have been suppressed using the transverse bunch-by-bunch $(\mathrm{BxB})$ feedback system. However other adverse effects of this instability, such as the vertical beam size increase along the bunch train cannot be cured by the feedback system. Therefore, to achieve the NSLS-II design current of $500 \mathrm{~mA}$ while maintaining a small vertical beam emittance, it is important to further understand the fast ion instability and develop mitigation techniques. This paper reports on a series of ion-instability observations at various fill patterns and beam currents using start-of-art NSLS-II diagnostic tools.

\section{INTRODUCTION}

The NSLS-II is an advanced third generation light source recently constructed at Brookhaven National Laboratory. With a relatively low dipole field of $0.4 \mathrm{~T}$, damping wigglers (DWs) are effectively used to decrease the horizontal emittance from $2 \mathrm{~nm}$.rad (bare lattice) to below $1 \mathrm{~nm}$.rad with $3 \mathrm{DWs}$ [1]. Emittance in the vertical plane has been measured as low as $4 \mathrm{pm}$.rad at low current. Vertical emittance during the present $250 \mathrm{~mA}$ operation is measured to be higher, but it is expected to be less in the future. A transverse bunch-by-bunch $(\mathrm{BxB})$ feedback has been implemented to cure bunch-to-bunch dipole motion caused by fast ion and other coupled bunch instabilities [2]. However, where the instabilities cause higher order modes of bunch motion, for example a quadrupole mode resulting in beam size blowup, BxB feedback is largely ineffective.

One such instability at NSLS-II is the fast ion instability. The theory of fast ion instability and early observations were reported almost 20 years ago [3-5]. As recent machine developments push for even lower emittances and higher currents, the instability is becoming more restrictive for modern light sources and colliders. More recent observations on newly constructed light sources and low emittance storage rings can be found for example in refs. [6-9].

This paper contains measurements related to resonant ion frequencies and ion-induced tune shifts. The ion frequency can be described by

$$
\omega_{i o n}=2 \pi f_{i o n}=\left[\frac{2 N_{b} r_{p} c}{A \sigma_{x, y}\left(\sigma_{x}+\sigma_{y}\right) T_{b}}\right]^{1 / 2},
$$

where $N_{b}$ is the number of electrons per bunch, $T_{b}$ is the bunch separation time, $r_{p}$ is the classical proton radius, $A$ is the atomic mass number of the ion, and $\sigma_{x, y}$ stands for the horizontal and vertical beam sizes. As is clear from Eq.(1), the ion frequency increases at higher bunch current and smaller beam size. Because beam sizes vary around the ring, and due to multiple residual ion species exist, there is typically a broad range of ion frequencies. Therefore, when ion-related instabilities occur, a fairly broadband set of unstable modes is often observed.

During a single pass of the electron bunch train, ions trapped in the beam potential will produce additional focusing on the electron bunch. The cumulative effect of ion focusing leads to a tune shift of the electron bunch. This effect is conveniently quantified with a kick factor due to ions, which, in the linear range of the ion potential, can be written

$$
\Delta Q_{y}=\frac{1}{4 \pi} \oint_{C} \beta_{y} K_{y}^{i o n} d s, \quad K_{y}^{i o n}=\frac{4 \lambda_{\text {ion }} r_{e} c}{\gamma \sigma_{y}\left(\sigma_{x}+\sigma_{y}\right)}
$$

where $\lambda_{\text {ion }}$ is the ion density and $r_{e}$ is the electron classical radius. Eqs. (2) are written for the vertical plane where the fast ion instability is typically dominant due to smaller emittance.

In the following sections, we present systematic experimental studies of ion-induced instabilities at NSLSII storage ring, including the observations at different vacuum conditions, chromaticity, coupling and insertion devices settings. We start by presenting instability observations at various vacuum conditions and beam current, followed by measurements at high chromaticity which helps to suppress ion-induced dipole motion. Measured tune shift along the bunch train clearly demonstrate that this effect is due to the presence of ions. We also present measurements of the lifetime of individual bunches which increases along the bunch train. This provides indirect but important evidence of ioninduced vertical beam size blow-up. Streak camera measurements are presented which allow one to clearly visualize the onset and the action of the instability along the bunch train. We conclude with a summary and plans for future studies. 


\section{IDENTIFICATION AND FEATURES OF FAST ION INSTABILITY AT NSLS-II}

Fast ion instability was first observed during the early stages of NSLS-II storage ring commissioning, when the vacuum conditions were fairly poor. The instability was detected as low as $\sim 10 \mathrm{~mA}$ of total beam current in 500 bunches. While the stored beam current during user operation has been gradually increased, thorough instability measurements have been carried out to track the changes. Some of these studies have been reported earlier $[2,10]$.

Top-off operation at NSLS-II was implemented in Oct 2015 and has run ever since. The second RF cavity was installed to allow for higher top-off current, which was gradually increased from $150 \mathrm{~mA}$ to $250 \mathrm{~mA}$. These steps have significantly increased the total accumulated beam dose (Amp.hours) and consequently improved the vacuum conditions which affect the fast ion instability.

As an example of earlier studies, we present experimental results from March 2015. These studies were performed at several values of average beam current. A single bunch train of 1000 consecutive bunches $(500 \mathrm{MHz} R$, bunch spacing $2 \mathrm{~ns}$, harmonic number 1320) was filled in the ring. Fill patterns were carefully controlled to minimize the bunch-to-bunch current variation.

With the transverse $\mathrm{BxB}$ feedback turned on, both horizontal and vertical instabilities were suppressed. There was no betatron motion sidebands observed in the button BPM spectra. With the feedback turned off, horizontal and vertical beam sizes measured by the x-ray pinhole camera increased, as shown in Fig. 1, which presents the data taken at $46 \mathrm{~mA}$. Specifically, when the horizontal feedback was turned off, the horizontal beam size jumped from $\sim 102 \mathrm{um}$ to $160 \mathrm{um}$ and the vertical beam size increased from $\sim 58 \mathrm{um}$ to $120 \mathrm{um}$. The beam sizes were fitted to a one-dimensional Gaussian function. Since the lattice coupling was relatively large at the early stage of machine commissioning, the $\mathrm{x}$-ray pinhole image was tilted due to coupling. When the horizontal feedback was turned off, the vertical beam size increased slightly, this is another indication of betatron coupling.

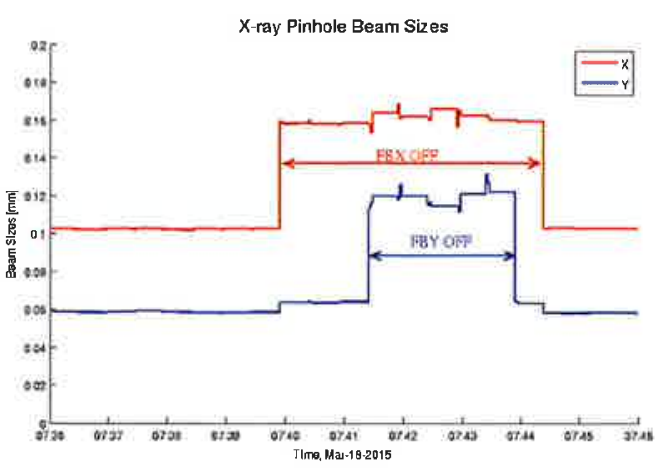

Figure 1: History plot of $\mathrm{x}$-ray pinhole camera measured beam sizes, with transverse $\mathrm{BxB}$ feedback ON/OFF, Beam was filled to $46 \mathrm{~mA}$ in 1000 bunches.

Simultaneously we performed instability grow-damp measurements, i.e. turn off the $\mathrm{B} \times \mathrm{B}$ feedback system for tens of $m$ s to allow the instability to grow to identify the unstable modes. Fig. 2 gives an example of these measurements using an FFT spectrum analyzer (SA) connected to a "hybrid delta" signal of two diagonal BPM buttons. Here vertical $\mathrm{BxB}$ feedback was switched off for $20 \mathrm{~ms}$. The FFT spectrum analyzer was externally triggered to be synchronous with the feedback gating signal. The center frequency of the spectrum analyzer was set to $F_{r f}+6 F_{r e v}$, where $F_{r f}$ and $F_{r e v}$ are the RF and revolution frequencies, to observe the most unstable mode of 1314 (or the complementary mode of -6 as the negative sideband is stronger at center frequency of $\left.F_{r f}+6 F_{r e v}\right)$.

Unstable modes are defined when a betatron sideband is seen at $p F_{r f}+m F_{r e v}+f_{x, y}$, where $p$ is an integer, $m=$ $0,1, \ldots 1319$ is the mode number and $f_{x} / f_{y}$ are betatron frequencies. Due to symmetry, the sideband at $p F_{\text {rf }}+$ $m F_{r e v}-f_{x, y}$ is sometimes called $-m$ mode. As can be seen, the vertical betatron frequency sidebands $(\sim 100 \mathrm{kHz}$ away from the center) started to grow until they were suppressed when the feedback was switched on again. The spectrum around $F_{r f}$ (mode 0 ) was also checked and no apparent betatron sidebands were detected during the period without the feedback. These results indicate that the instability with the fastest growth rate was not due to the resistive wall, which typically causes lower frequency instabilities with the unstable mode -1 .

Additional insight can be gained by analyzing the bunch-by-bunch position data on every turn, recorded by the feedback system digitizer during the grow-damp measurement. An indication of an ion-induced instability is that unstable modes depend on the stored beam current, see Eq. (1). Due to beam size variation around the ring, a typical ion-induced instability has a broadband hump of unstable modes. Fig. 3 illustrates the unstable modes measured at $17 \mathrm{~mA}, 31 \mathrm{~mA}$ and $96 \mathrm{~mA}$. As the total beam current is increased, the ion frequencies grow and the unstable modes tend to shift away from the $F_{r f}$ frequency. 


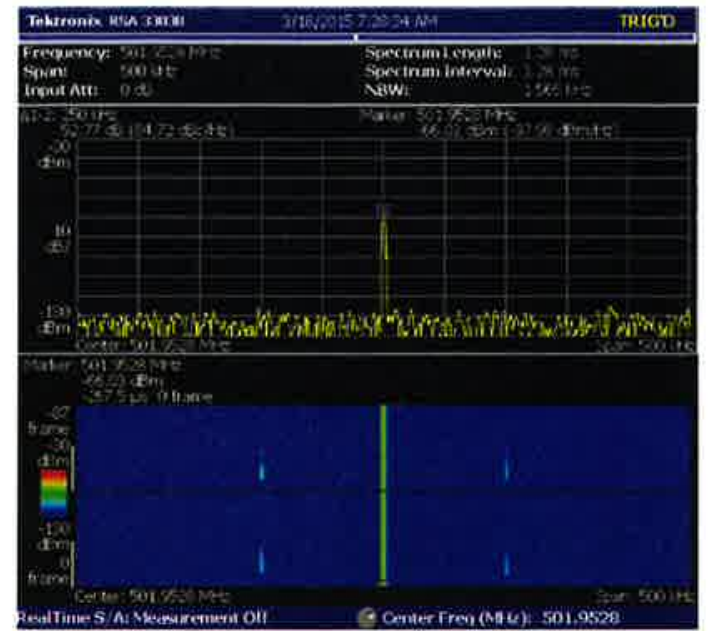

Figure 2: FFT spectrum while vertical BxB feedback was gated OFF for $20 \mathrm{~ms}$. Center frequency of the spectrum analyzer was at $F_{r f}+6 F_{\text {rev. }}$. Vertical betatron sidebands appear around $100 \mathrm{kHz}$ and are suppressed once the feedback was switched back ON.
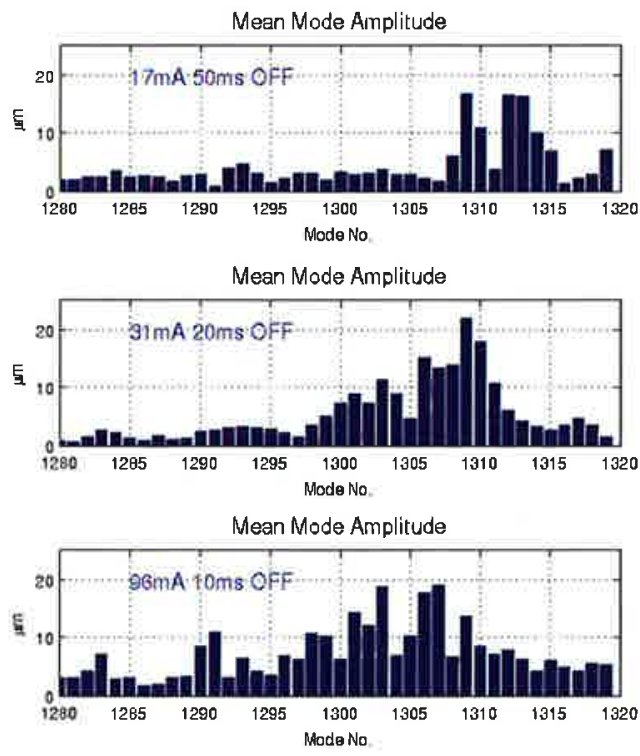

Figure 3: Average unstable mode amplitudes during the feedback OFF period at different total beam currents. The ring was filled with $\sim 1000$ bunches evenly distributed. $\mathrm{B} \times \mathrm{B}$ feedback was switched OFF for short period of time to allow the most unstable modes grow.

It is worthwhile to point out that Figs. 1-3 were measured in March 2015, when the machine vacuum was relatively poor. After months of high current operation, storage ring vacuum condition keeps improving. At the same $100 \mathrm{~mA} 80 \%$ fill ( 1000 bunches), average vacuum pressure improved from $3 \mathrm{nTorr}$ (Mar 2015) to $2.2 \mathrm{nTorr}$ (Aug 2015), 2.0nTorr (Apr 2016) and 1.8nTorr (Aug 2016).

More recently, in April 2016, a similar study was repeated. Two distinct fill patterns were tried, one with a single bunch train of 1000 consecutive bunches, the same pattern used in March 2015 and for user operations. The second fill pattern had two bunch trains with 500 consecutive bunches each and 160 empty buckets inbetween. In order to see the difference between a single train and two-train fills, the total beam current were kept roughly the same. Specifically, we filled to $56 \mathrm{~mA}$, $114 \mathrm{~mA}, 169 \mathrm{~mA}, 225 \mathrm{~mA}$ and $270 \mathrm{~mA}$. Bunch-to-bunch current variation was within $10 \%$ RMS not counting the rising/falling edge bunches.

For illustration, Fig. 4 plots the measured fill pattern at $267 \mathrm{~mA}$ with one train of 1000 bunches. A single bunch was filled in the ion gap at bucket $\# 1200$ for continuous tune measurement. Details on how the fill pattern is measured can be found in [11]. In contrast to the studies in March 2015, the rising and falling edges of the bunch trains were not trimmed. We found the edge bunches have little effect on the ion-induced instability.

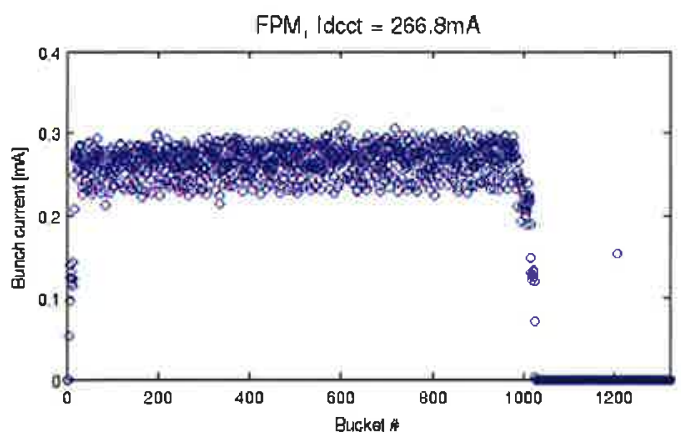

Figure 4: Bunch to bunch current with 1000 bunches filled to $267 \mathrm{~mA}$, bunch to bunch current variation due to the uneven fill pattern from injector.

Fig. 5 gives an example for the case of $268 \mathrm{~mA} / 1000$ bunch fill and all insertion device gaps closed. The vertical feedback was turned off for $10 \mathrm{~ms}$ while the horizontal feedback was on all the time. From the turnby-turn positional information, the RMS motion of individual bunches along the bunch train is plotted at the top. As can been seen, the vertical oscillations were growing along the bunch train, which is the expected behavior of the fast ion instability. For two bunch-train fills, the head bunches within each train have less RMS motion than the tail bunches, also consistent with the fast ion instability.

Mode analysis shows many unstable modes around the frequency $1280^{*} F_{\text {rev }}$ (or $-40 F_{\text {rev }}$ ). The bottom plot in figure 5 shows the two most unstable modes, 1279 and 1280 that are caused by the fast ion instability. The figure also shows another pair of modes, 1318 and 1319 which are driven by resistive wall impedance.

Clearly, ion-induced modes have a much faster growth rate but they tend to saturate early. On the other hand, the resistive wall instability develops slower, but maintains exponential growth long enough to overtake the fast ion instability (provided that the $\mathrm{BxB}$ feedback is off long enough). 
Note that the relative strength of the fast ion and resistive wall instabilities depends on the machine state, i.e. the number of closed IVU gaps, vacuum conditions, fill pattern, bunch length, etc. In general, as more IVUs are installed in the ring, the resistive wall impedance becomes stronger, while ion-related effects are expected to be weaker with further vacuum conditioning.
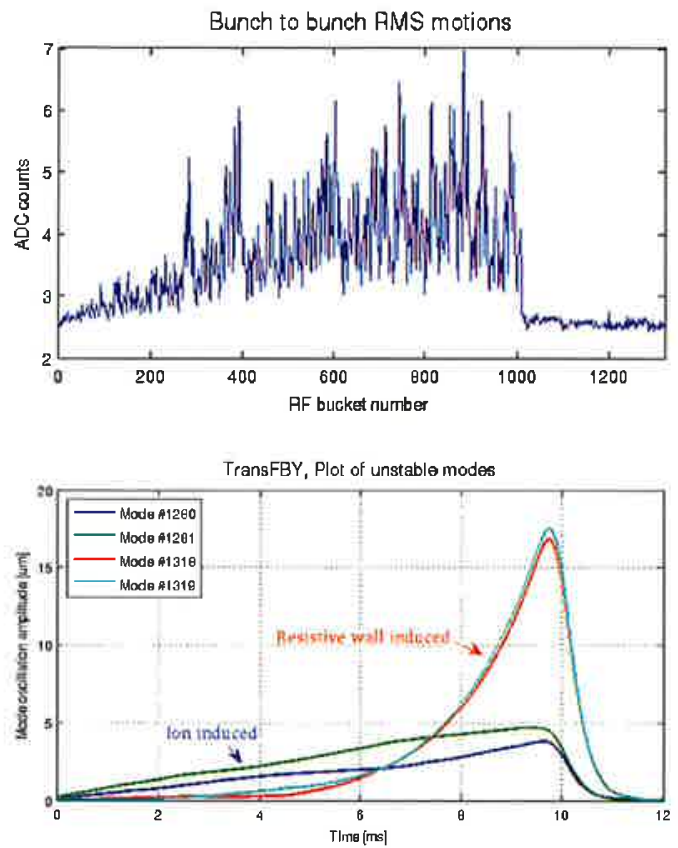

Figure 5: (Upper) Bunch-to-bunch RMS motions along the 1000 -bunch train with vertical feedback off for $10 \mathrm{~ms}$. Beam was filled to $268 \mathrm{~mA}$ with all the insertion device gaps open. (Bottom) Time domain plot showing the most unstable modes fast ion modes (\# 1280-1281) and resistive wall instabilities (mode \# 1318-19, sometimes referred to as mode nubmers $-1,-2$ ).

\section{CHROMATICITY EFFECT}

During studies with high chromaticity of $+7 /+7$ [12], up to $100 \mathrm{~mA}$ of beam current was stored in the ring without BxB feedback. No instabilities were evident from BPM turn-by-turn data, which could only provide the information about the lowest unstable mode $(0$ or -1$)$. To check whether higher order unstable modes are present, an FFT spectrum analyzer was configured to measure the broadband button BPM spectrum. Unstable modes were observed at $100 \mathrm{~mA} 80 \%$ fill. Among these the most unstable modes were $\mathbf{- 8}$ horizontally (stronger sideband at $p^{*} F_{r f}+8^{*} F_{r e v}-f_{x}$, which is the same sideband as $p^{*} F_{r f}+$ $1312 * F_{r e v}+f_{x}$ ) and -12 vertically (stronger sideband at $p^{*} F_{r f}+12^{*} F_{r e v}-f_{y}$ or equivalently at $p^{*} F_{r f}+1308^{*} F_{r e v}+$ $f_{y}$ ).

Similar to Fig. 3, our analysis of BxB feedback data has identified the most unstable modes at high chromaticity. Fig. 6 compares the unstable modes at different currents and filling patterns. The top two plots have the same
1000 -bunch $(80 \%)$ fill pattern but at a different total current, as indicated in the legend. As the beam current roughly doubled, the most unstable mode shifted from -8 to -12 which agrees with Eq. (1) for the resonant ion frequency. The third plot shows the result for the $100 \mathrm{~mA}$ $50 \%$ fill ( 660 bunches). Compared to the middle plot with the same total current, the ion frequency has shifted to even higher values (the most unstable mode is at -15 ), which is consistent with $60 \%$ higher intensity per bunch.
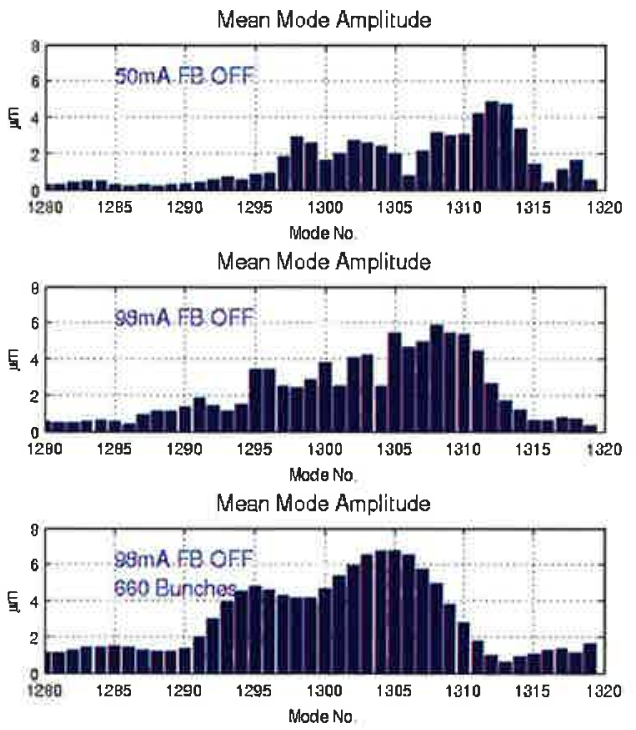

Figure 6: Average unstable mode amplitudes measured for chromaticity $+7 /+7$ lattice. BxB feedback was off, there were ion induced unstable modes observed.

During a more recent study in August 2016, 250mA beam was filled into the ring with the chromaticity $+7 /+7$, to be compared with the nominal $+2 /+2$ chromaticity lattice during user operation. To identify the dominant unstable mode the feedback was turned off for $20 \mathrm{~ms}$ for the high chromaticity case and for $10 \mathrm{~ms}$ for the nominal case. Fig. 7 compares unstable mode amplitudes, averaged over the last $2 \mathrm{~ms}$ of the feedback-off period. Even though in both cases the locations of the most unstable modes are similar, i.e. a broadband hump with mode indexes 1220-1300, the amplitude for the high chromaticity case is roughly an order of magnitude lower. This is a qualitative comparison as the feedback was switched off for different period of time. Nevertheless, the fast ion instability is still observable even at high chromaticity.

With nominal chromaticity, the resistive wall induced instability (mode \#1318, 1319) can be observed. No unstable resistive wall mode was detected at high chromaticity likely because the growth rate is smaller than Landau damping rate. 


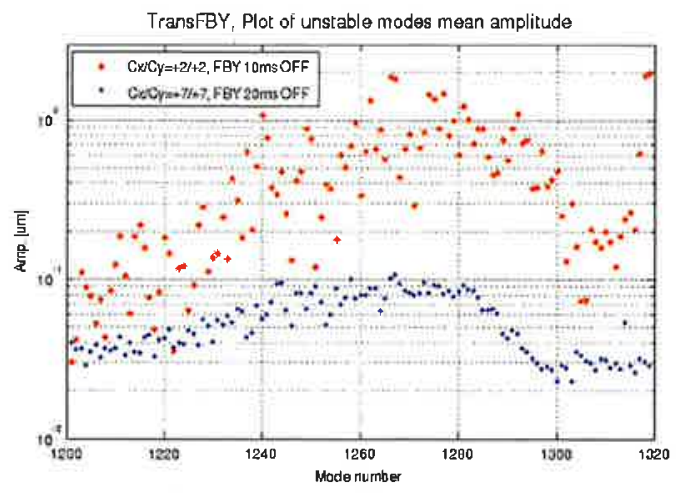

Figure 7: Unstable mode amplitudes, averaged over the last $2 \mathrm{~ms}$ of the feedback off period for the cases of high chromaticity $(+7 /+7)$ and nominal chromaticity $(+2 /+2)$. $\mathrm{BxB}$ feedback was turned off for $20 \mathrm{~ms}$ and $10 \mathrm{~ms}$ for the cases of high and nominal chromaticity, respectively.

\section{BETATRON TUNE SHIFT}

To investigate the fast ion focusing effect along the bunch train, the single bunch spectrum was first measured to determine the bunch betatron oscillation frequency and amplitude. We used two different methods to determine the bunch-by-bunch tune with little disturbance to the beam. With the first method, BxB feedback was turned off for the particular bunch being measured while keeping the rest of bunches stable with feedback. At high current operation, the "feedback off bunch" would typically become unstable and exhibit betatron motion, while at low current the bunch was externally driven by sweeping frequency near the betatron frequency. In both cases the betatron motion was analyzed to extract the tune and oscillation amplitude. Repeating this measurement for every bunch gives the bunch-by-bunch betatron oscillation frequency and amplitude.

The second method kept the feedback on for all the bunches. This method takes advantage of the fact that as the feedback tries to suppress the motion near the betatron frequency, a notch was created in the bunch spectrum at the incoherent tune of the bunch. This allows us to infer this notch as the betatron tune of the bunch. A similar method has been used to measure the betatron at other facilities [13].

Figure 8 gives the result of the bunch-by-bunch spectrum in the vertical plane during $250 \mathrm{~mA}$ top-off operation. There were $\sim 1000$ bunches filled with all insertion device gaps closed. The top plot shows the results of the first method, with $\mathrm{BxB}$ feedback switched off one bunch at a time, from bucket 10 to 1000 , in 10 bucket steps. A small excitation was applied to better detect the betatron peak, which was necessary for bunches at the head of the train where ion-induced oscillations are too weak to be measured.
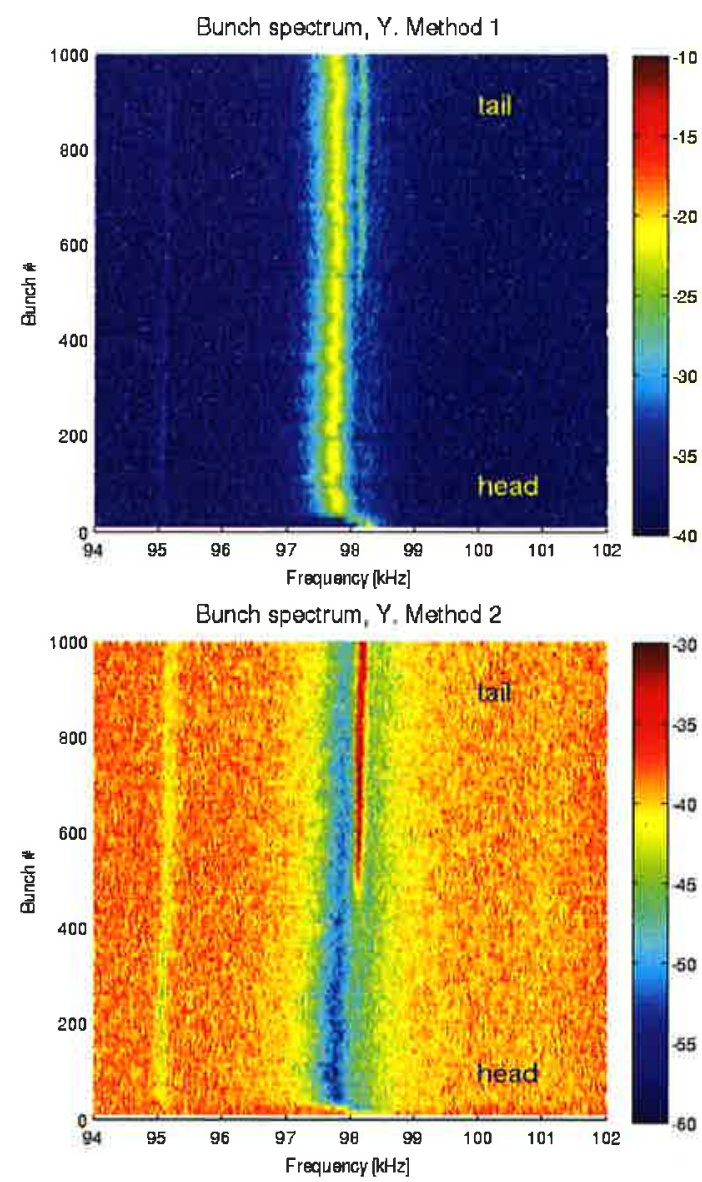

Averaged bunch spectrum, $Y$

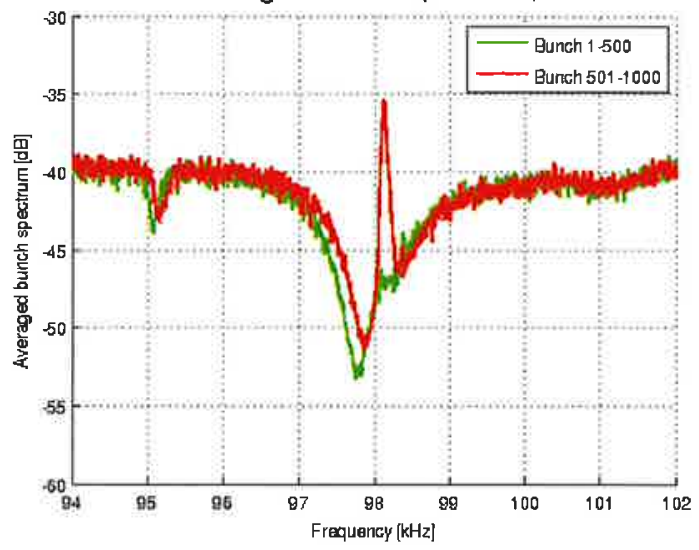

Figure 8: Single bunch vertical tune and amplitude measurement along a 1000 -bunch train during $250 \mathrm{~mA}$ top off operation. (top) $\mathrm{BxB}$ feedback switched off one bunch at a time to see the betatron motion of each particular bunch; (middle) $\mathrm{BxB}$ feedback on and betatron tune measured from the dip on the bunch spectrum; (bottom) Averaged bunch spectrum with feedback on.

The middle plot is obtained using the second method measuring the same buckets from 10 to 1000 in 10 bucket steps. In this case the feedback was always on, and a spectral dip was observed near the betatron frequency (and at the $+/-\mathrm{fs}$ sidebands). Both methods confirmed the 
emergence of a second peak near the betatron frequency only for bunches after \#500. This peak is due to ion accumulation along the bunch train.

The averaged bunch spectrum with feedback on is shown in the bottom plot. Again an ion-induced peak is clearly visible just above of the betatron dip in the spectrum, but it only appears for bunches after bucket \#500. Similar measurements were carried out earlier when vacuum conditions were not as good. What we observed then was that the second peak appeared earlier in the train. For example in Oct 2015 measurement with $150 \mathrm{~mA}$, the peak appeared at bunch \#300. By splitting the train in to two or four bunch trains, the ion induced peak was not as obvious.

From the Fig. 8 spectra, one can see the vertical betatron frequency increases slightly from the head to the tail of the bunch train. A measurement by scanning the bunches typically takes about 10 minutes. To eliminate the possibility that the observed tune shift along the bunch train is caused by machine drifts, it is desired to measure the tunes of individual bunches at the same time. The feedback digitizer is capable of saving $\sim 9500$ turns of data for all 1320 buckets. Least squares fitting was applied to the bunch spectrum, hence accurate tunes can be measured for different bunches in the train.
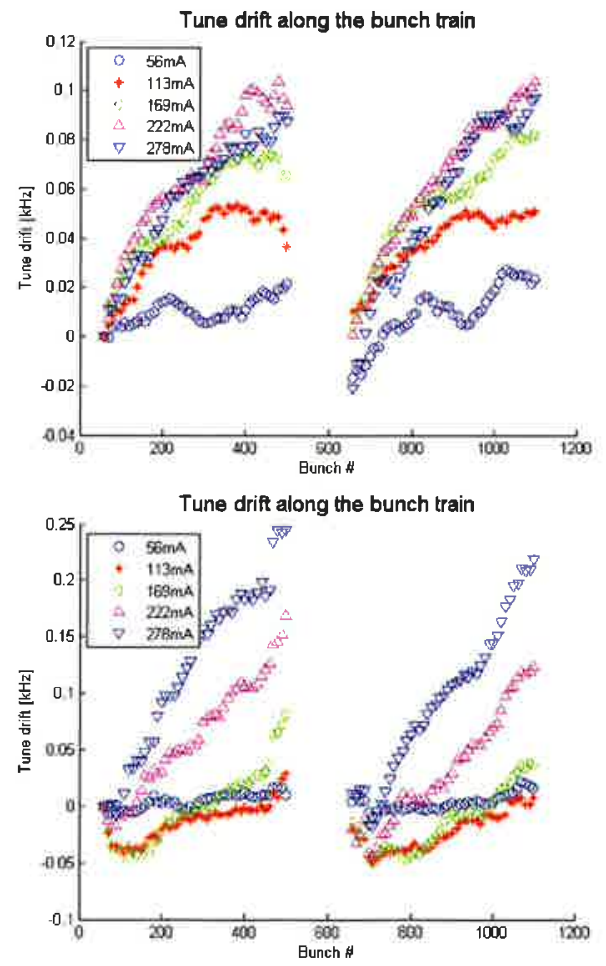

Figure 9: fx/fy (top/bottom) shift along the bunch train at various total beam currents.

Precise tune measurement along the bunch train was investigated. With two 500-bunch trains in the machine separated by 160 empty buckets, individual bunch tunes were simultaneously measured by fitting the dip at the betatron frequency for each bunch.

Figure 9 gives an example of accurate measurements of horizontal and vertical tune shifts along the bunch train obtained from 9500-turn bunch-by-bunch data. Except for the rising and falling edges, the bunch train was fairly uniform with bunch-to-bunch current variation within $20 \%$. Bunch-to-bunch tune was fitted from the single bunch spectrum similar to the one plotted in Fig 8 . It is clear that the betatron frequency increases along the bunch train in both planes and that the slope is bigger at higher total beam current. We believe the horizontal tune shift along the bunch train is due to ion accumulation as well. It is also clear that a 160 bucket long gap between the bunch trains is adequate to cure the ion-related effects and reduce the instability-induced motion.

We also repeated the measurement for a 1000-bunch train fill, and observed a similar bunch-to-bunch tune shift along the train. Compared to the two train fill as shown in Fig. 9, where the maximum tune shift was $\sim 0.25 \mathrm{kHz}$ vertically with total beam current of $278 \mathrm{~mA}$, the tail bunches in the 1000-bunch train had tune shift $\sim 0.4 \mathrm{kHz}$ at $270 \mathrm{~mA}$. The varying tune-shift along the train clearly indicates the trapped ion density increase along the train.

In order to optimize the filling pattern, and determine how long of an ion gap is needed between trains, a probe bunch was filled at different locations after a bunch train. The method is similar to the one reported in [14] to probe electron cloud effects. Two different fills were tested. In the first fill, 1000 bunches were filled into a singe bunch train with a total beam current of $170 \mathrm{~mA}$. For the second fill, 500 bunches were filled in one train to a total beam current of $180 \mathrm{~mA}$. The rising and falling edges of the bunch train were trimmed to have a sharp rectangular fill pattern. The BxB feedback was turned on for all bunches in the train, to suppress dipole instabilities. A probe bunch of $0.1 \mathrm{~mA}$ was placed at discrete buckets behind the train. The probe bunch had no feedback acting on it, so residual ions drove this bunch unstable. Recording the TbT motion of the probe bunch with the $\mathrm{BxB}$ feedback digitizer we were able to extract the oscillation amplitude which is shown in Fig. 10 as a function of the distance behind the main train.

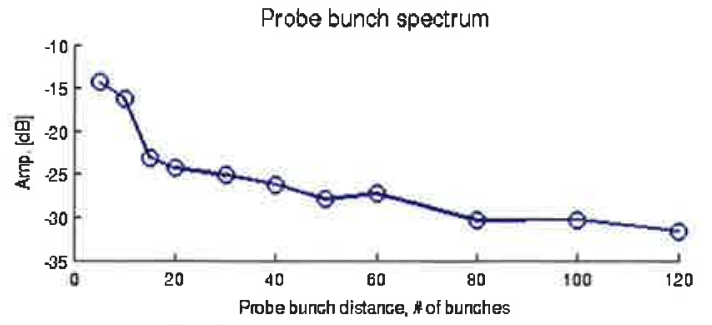

Figure 10: Probe bunch vertical dipole motion amplitude vs. distance from the main bunch train with $170 \mathrm{~mA}$ in 1000 bunches.

Fig. 10 gives an indirect measurement of ion density after the bunch train. The noise floor of the single bunch 
spectrum is around $-35 \mathrm{~dB}$. The probe bunch oscillation amplitude decreases rapidly with more than 10 empty buckets from the main train and approaches to the noise floor level after 80 empty buckets. This suggests that most of the ions can be cleared with 80 empty buckets and also explains the tune drift in two trains in Fig. 9.

\section{LIFETIME OF INDIVIDUAL BUNCHES}

At the NSLS-II storage ring the bunch-by-bunch current can be measured by the 4-button BPM sum signal [11]. Fitting the decay of individual bunch current one can calculate its lifetime. If the bunches in the train are identical, one would expect no difference in individual bunch lifetimes. On the other hand, if there is a variation in current between the bunches, the lifetimes should reflect this since the Touschek scattering rate is proportional to the bunch current. Due to imperfections of the NSLS-II injector, the storage ring fill pattern is not uniform, as shown at the top of Fig. 11. The main bunch train consists of $\sim 1050$ bunches, with the first 800 having a roughly $50 \%$ more charge per bunch than the rest of the train. A smaller periodic variation in charge per bunch (again coming from the injector) is also clearly visible.
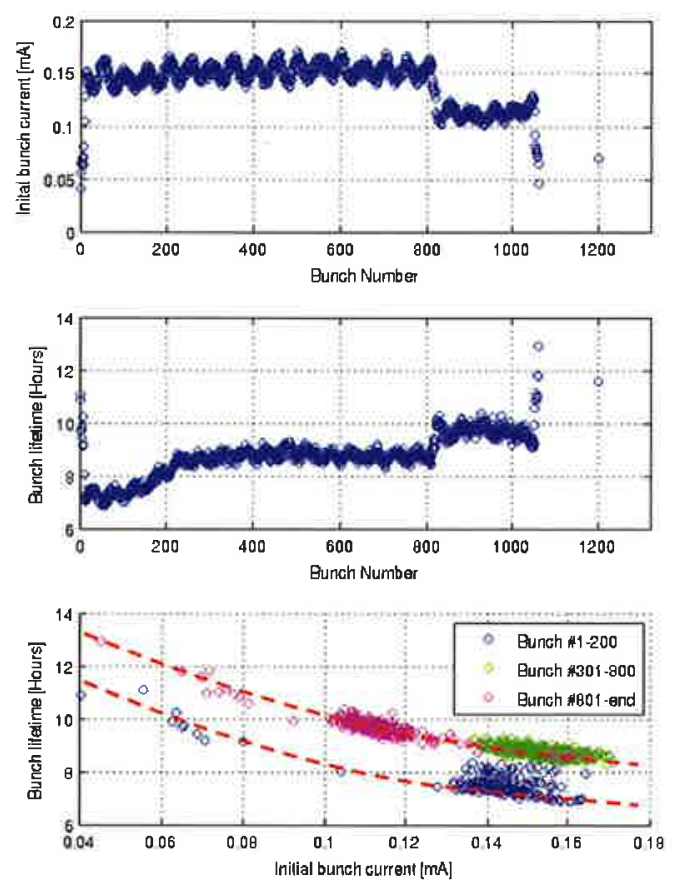

Figure 11: Lifetime of individual bunches obtained from fill pattern monitor data. (top) Initial fill pattern, note a camshaft bunch filled at bucket \#1200, total beam current $150 \mathrm{~mA}$. (middle) Lifetime vs. bucket number. (bottom) lifetime vs. initial current per bunch.

This fill pattern was a typical one when NSLS-II was running in decay mode (no top off). The storage ring was initially filled to $150 \mathrm{~mA}$. Bunch to bunch currents were recorded with 1 minute intervals. Data taken over 60minutes was linearly fitted to obtain individual bunch lifetimes. As one would expect, the lifetimes, plotted in the middle of Fig. 11, inversely correlate with charge per bunch. For instance the tail portion of the train, starting with bunches around bucket 800 , has a roughly $30 \%$ longer lifetime. Note that we do not expect a perfect inverse correlation between lifetime and current because, unlike the Touschek lifetime, the gas-scattering component of beam losses does not depend on the bunch charge.

It is instructive to re-plot the same individual bunch lifetimes as a function of the initial single bunch current; this plot is shown in Fig. 11 bottom. The lifetimes clearly fall into two distinct bands, the shorter one corresponds to the first 200 or so bunches of the train, while the longer lifetime band contains the remainder of the train. Even though the intensity per bunch is not different, the first 200 bunches have significantly lower lifetimes than the following 600 bunches. We believe the most likely explanation of longer lifetimes in the second band is the ion-induced blow-up in the vertical beam size, which occurs in bunches after \#200. The BxB feedback system was on during these measurements $(150 \mathrm{~mA}$ user operations), so the dipolar beam motion was fully suppressed.

It would be valuable to directly measure the vertical beam size blowup along the train. Unfortunately, there is presently no tool at the NSLS-II ring to measure transverse size of individual bunches along the train. Even if we could gate out the light from individual bunches, the visible diagnostic beamline lacks the resolution to measure the vertical beam size, while the $x$-ray pinhole beamline does not have sufficient intensity to measure the bunches turn-by-turn. Presently, for a reliable beam size measurement, the pinhole camera typically uses $100 \mathrm{~ms}$ exposure time or about $38 \mathrm{k}$ turns. With the future addition of the second $\mathrm{x}$-ray pinhole beamline we are planning to use a gated camera which should make the bunch-bybunch profile measurement feasible. Alternatively, we may try the new technique that combines a double slit interferometer with a fast-gated camera [15]. In the meantime, to prove that the lifetime increase along the bunch train is due to the vertical beam size increase along the train, we have measured the $\mathrm{x}$-ray pinhole beam sizes for different length bunch trains.

First X-ray pinhole camera measured beam sizes have been compared between one train ( 1000 bunches with 320 empty buckets) and two trains (500 bunches per train with 160 empty buckets in between). It was clear that with the two bunch train fill, the measured vertical beam size was smaller compared to the one-train fill, which is what one would expect for a blow-up caused by ion instabilities.

To further investigate the beam size blowup due to ions, we measured transverse beam size for different length bunch trains while maintaining constant current per bunch. The beam was initially filled into a 1000-bunch train with the total current of $143 \mathrm{~mA}(\sim 0.14 \mathrm{~mA}$ per bunch). Transverse beam sizes were measured with the Xray pinhole camera, and then the tail 100 bunches were 
knocked out using the bunch cleaning feature of the $\mathrm{B} \times \mathrm{B}$ feedback system [2]. The procedure was repeated until the train length reached 200 bunches. The measurement results are presented in Fig 12 . We notice a substantial increase in the vertical beam size when the train length exceeds 400 bunches, while the horizontal beam size basically does not change.

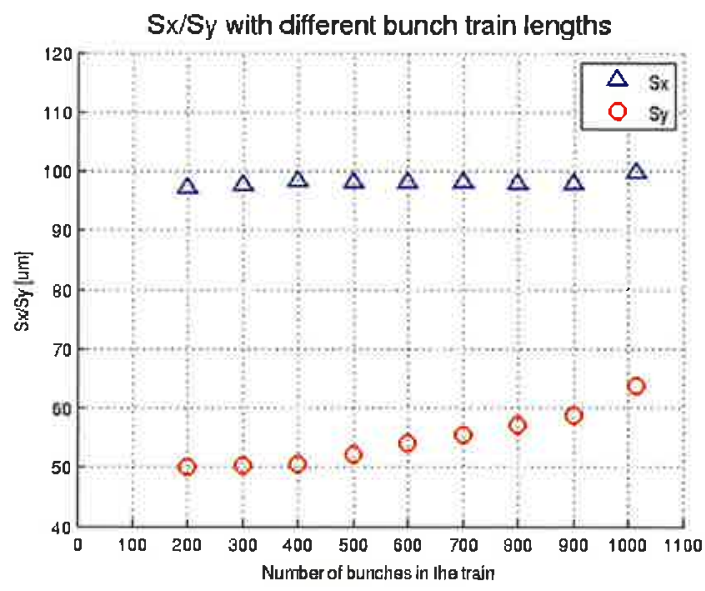

Figure 12: X-ray pinhole measured beam sizes for filled trains of different length.

\section{STREAK CAMERA OBSERVATIONS}

NSLS-II uses a Hamamatsu C5680 streak camera for longitudinal profile measurements. The camera is setup to accept visible synchrotron radiation from a cell 30 bending magnet. The SLM (synchrotron light monitor) diagnostic beamline was constructed to guide the dipole radiation to the experimental hutch where various camera setups are available.
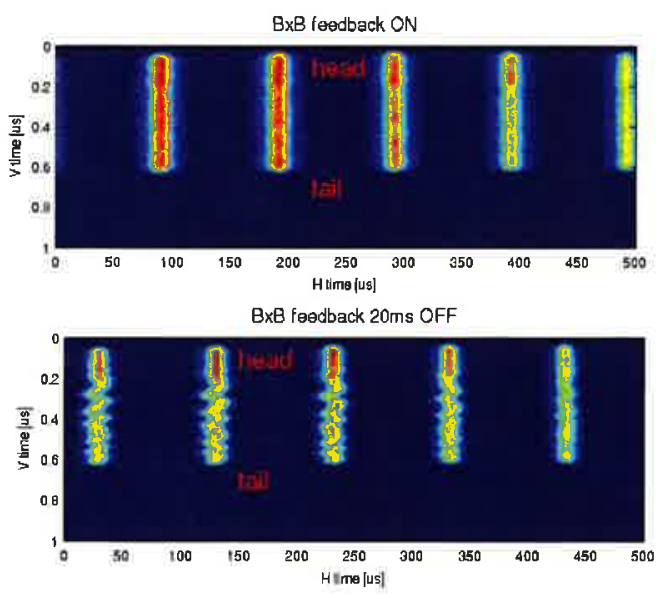

Figure 13: Dual sweep streak camera image using a slow sweep module. (Top) with BxB feedback on, there was no instability observed in the streak camera $y-z$ image. (Bottom) with the feedback turned off for $20 \mathrm{~ms}$, and the streak camera trigger delay adjusted $19 \mathrm{~ms}$ after the feedback off, bunches after \#100 show vertical motions on the streak camera image.
The streak camera can be configured to measure the vertical-longitudinal $(y-z)$ beam profile, which is useful when single- and multi-bunch instabilities are present [16]. With the Hamamatsu M5677 slow sweep plug-in module, the streak camera can see the $y-z$ image of bunches along the bunch train. It is worthwhile to point out that the diffraction limit of SLM beamline is $\sim 60 \mu \mathrm{m}$, while the nominal vertical beam size in that location is $\sim 10 \mu \mathrm{m}$. Therefore it is impossible to accurately measure the vertical beam size directly from the streak camera image, however the latter is very useful to observe instability-induced vertical dipolar beam motion.

A series of experiments were done and streak camera $(y-z)$ images were recorded with different length bunch trains stored in the ring (one train at a time). When the $\mathrm{BxB}$ feedback was on, there were no vertical dipole instabilities and the turn-by-turn $(y-z)$ images were similar to those shown in Fig. 13 upper plot.

For this particular case, the ring was filled with a 300 bunch continuous train with total current $\sim 63 \mathrm{~mA}$, which gives roughly $0.2 \mathrm{~mA}$ per bunch. The streak camera was operating in dual sweep mode with the vertical sweep time set to $2 \mu \mathrm{s}$ (which is close to the revolution period of $2.64 \mu \mathrm{s}$, to record one turn profiles of stored bunches. In the plot, vertical scale was adjusted to $1 \mu$ s to see the where the 300 bunches exists) and the slow sweep clock was set to $10 \mathrm{kHz}$. The slow (horizontal) sweep time was set to $500 \mu$ s to see the bunch train during five turns with $100 \mu$ s separation. Each vertical strip in the figure is a one-turn image with head bunches at the top.

To see the fast ion induced dipole motion, the vertical $\mathrm{BxB}$ feedback was gated off for $20 \mathrm{~ms}$ and the streak camera horizontal trigger delay was adjusted to $19 \mathrm{~ms}$ after the feedback was turned off. As shown in the bottom image, the head bunches were still stable while the tail bunches (rougly bunch \#100 and beyond) were executing significant dipole motion. This is in agreement with the $\mathrm{BxB}$ digitizer positional measurements or the fast ion instability presented earlier in this paper.

\section{SUMMARY}

Fast ion instability has been observed in the NSLS-II storage ring at different vacuum conditions, beam currents, filling patterns, chromaticities and insertion device settings. Two signature features of fast ion instability are always present. First, unstable modes move up in frequency at higher bunch currents. Second, these modes tend to have a broadband 'hump' spectral feature, presumably due to the beam size (and hence the corresponding ion frequency) variation around the ring, as well as due to multiple ion species involved in the instability.

We have studied the bunch-to-bunch tune shift, lifetime increase along the bunch train, ion intensity with a probe bunch in the gap, and vertical oscillation developing along the bunch train captured with streak camera. All these measurements show that the fast ion instability is a 
dominant instability in the NSLS-II storage ring. Even with further vacuum conditioning, we believe the fast ion instability, if left unaddressed, will remain strong when we reach the full design current of $500 \mathrm{~mA}$ and may prevent reaching the design vertical emittance of 8 pm.rad. Further studies will be helpful to characterize the instability at higher beam current and with more insertion devices. Optimization of the storage ring fill pattern, the bunch-by-bunch feedback settings and vacuum conditioning will be necessary to achieve the design emittance.

We thank Bel Bacha for support on hardware setups; Dmitry Teytelman for fruitful discussions; Om Singh and Timur Shaftan for continuous support on machine studies.

\section{REFERENCES}

[1] F. Willeke, "Commissioning of NSLS-II", IPAC'2015

[2] W. Cheng, et al., "Commissioning of BxB feedback System for NSLS-II Storage Ring", IBIC'2014

[3] T. Raubenheimer, F. Zimmermann, "Fast beam-ion instability I. Linear theory and simulations", Phys. Rev. E52, No. 5, 5487 (1995)

[4] G.Stupakov, T. Raubenheimer, F. Zimmermann, "Fast beam-ion instability II. Effect of ion decoherence", Phys, Rev, E52, 5499 (1995)

[5] J. Byrd, et al., "First Observations of a Fast Beam-Ion Instability", Phys. Rev. Lett. 79, 79 (1997)

[6] A. Chatterjee, et al., "Fast ion instability at the Cornell Electron Storage Ring Test Accelerator", PRST-AB, 18, 064402 (2015)

[7] L. Wang, et al., "Beam ion instability: Measurement, analysis, and simulation", PRST-AB, 16, 104402 (2013)

[8] B. Jiang, et al., "Investigation of fast ion instability in SSRF", Nulc. Inst. Meth. A, Vol. 614, 331 (2010)

[9] R. Nagaoka, et al., "Fast Beam-ion Instability Observations at SOLEIL", IPAC'2010

[10] W. Cheng, et al., "Observation of Ion-induced Instabilities at NSLS-II Storage Ring”, IBIC'2015

[11] W. Cheng, et al., "NSLS-II Fill Pattern Monitor and Control", IBIC'2015.

[12] Y. Li, L. Yang, "Multi-objective dynamic aperture optimization for storage rings", Int. J. Mod. Phys. A 31,1644019 (2016)

[13] C.H. Kuo, et al., "Operation Status of Bunch-bybunch Feedback System for the TLS", IPAC'2011

[14] T. Ieiri, et al., "Bunch-by-bunch measurements of the betatron tune and the synchronous phase and their applications to beam dynamics at KEKB", PRSTAB, 5, 094402 (2002)

[15] S.T. Wang, R. Holtzapple, D.L.Rubin, "Single-shot beam size measurements using visible-light interferometry at CESR" Nucl. Inst. Meth. A, Vol. 847, 34-41 (2017)

[16] W. Cheng et al., "Longitudinal Bunch Profile Measurement at NSLS-II Storage Ring”, IBIC'2015. 